\title{
Efficacy of Adding Dexmedetomidine in Fascia Iliaca Compartment Block to Provide Analgesia for Positioning Femur Fracture Patients before Spinal Anesthesia
}

\author{
Mohamed Abd-Allah Amin, ${ }^{1}$ MD; Abdalla M Abdalla, ${ }^{2}$ MD; Sabri A Soltan, ${ }^{2}$ MD.
}

\section{* Corresponding Author: \\ Mohamed Abd-Allah Amin drmaa73@gmail.com}

\section{Received for publication September 27, 2020; Accepted October 12, 2020; Published online October 12, 2020.}

\section{Copyright 2020 The Authors published by Al-Azhar} University, Faculty of Medicine, Cairo, Egypt. All rights reserved. This an open-access article distributed under the legal terms, where it is permissible to download and share the work provided it is properly cited. The work cannot be changed in any way or used commercially.

doi: 10.21608/aimj.2020.44250.1328.

${ }^{1}$ Anesthesia and intensive care Department, Faculty of Medicine for Girls, Al-Azhar University.

${ }^{2}$ Anesthesia and intensive care Department, Faculty of Medicine, Al-Azhar University.

\begin{abstract}
Background: Fascia iliaca compartment block (FICB) has been used to provide analgesia and facilitate positioning for spinal anesthesia in fracture femur patients with the problem of delayed onset of action.

Aim of the study: The current study aimed to assess effectiveness of dexmedetomidine as adjuvant to FICB with bupivacaine to facilitate positioning for spinal anesthesia in fracture femur patients with decrease time to sensory block as primary outcome.

Patient and Methods: In this prospective double blinded randomized controlled study, 60 patients, ASA I-III, aged 50-70 years, underwent femur fracture surgeries under spinal anesthesia were included. Patients were randomly assigned into two equal groups according to drug injected in FICB; Control group received $40 \mathrm{ml} 0.25 \%$ bupivacaine, and Dexmedetomidine group received dexmedetomidine $80 \mu \mathrm{g}$ in $40 \mathrm{ml}$ $0.25 \%$ bupivacaine. Twenty minutes after FICB, spinal anesthesia was given. Time to sensory block, fentanyl requirements, and pain during positioning were compared. Postoperative; time to first analgesic request, pain score, and total analgesic requirements were compared.

Results: Time to sensory block was significantly shorter and pain during positioning for spinal anesthesia was significantly lower in Dexmedetomidine group, but there were no differences regarding fentanyl requirements. Postoperatively, Pain score was significantly lower after 6 and 8 hours in Dexmedetomidine group, time to first analgesic request was longer, and total analgesic requirements was less in Dexmedetomidine group.

Conclusion: Addition of dexmedetomidine to bupivacaine in FICB is effective in enhancing onset and decreasing severity of pain during positioning for spinal anesthesia in fracture femur patients, with added postoperative analgesic effect.
\end{abstract}

Keywords: Dexmedetomidine; fascia iliaca; fracture femur; positioning; spinal.

Disclosure: The authors have no financial interest to declare in relation to the content of this article. The Article Processing Charge was paid for by the authors.

Authorship: All authors have a substantial contribution to the article

\section{INTRODUCTION}

Femur fractures are commonly seen in elderly, elderly people are at a higher risk of developing low energy proximal femur fractures due to osteoporotic bone changes, and increased risk of falls. ${ }^{1}$ Due to increased aging, femur fractures are predicted to rise worldwide and results in more admissions to orthopedic trauma surgical units. ${ }^{2}$

Current preoperative pain relief for those patients is poor. Intravenous analgesics (Paracetamol, nonsteroidal anti-inflammatory drugs, and opioids) are the most frequently used but have their limitations like, systemic side effects, maybe contraindicated due to their interactions with other drugs, and may be ineffective for dynamic pain which patients are likely to suffer during movement. Additionally, patients reported inadequate analgesia for this severe painful fracture with up to $40 \%$ of patients did not receive any analgesics. ${ }^{2,3}$

Poor pre-operative pain management in patients with femur bone fractures may be harmful, it can initiate physiological stress response which may lead to tachycardia, hypertension and arrythmias (may harm elderly and cardiac patients), an increase in the hemostatic system, deep venous thrombosis resulting from venous stasis, impaired immune system which result in increased infections, postoperative fatigue and delay in return of muscle function. ${ }^{4}$ 
Attempts for creating effective pre-operative analgesia and facilitating positioning for spinal anesthesia have led to design of various methods for local anesthesia. Peripheral nerve block is highly considered due to its effective role, added postoperative analgesic effect, and patient movement enhancement. $^{4}$

When considering preoperative regional analgesia or postoperative pain management for femur fracture surgeries, there are numerous different approaches, such as paravertebral block, fascia iliaca compartment block (FICB), femoral nerve block (FNB), sciatic nerve block, and epidural analgesia. One of these block methods, which is currently considered in orthopedic surgery is FICB, it is easily performed, done in supine position with no need for patient movement, and can be performed outside the operation room. ${ }^{4}$

Fascia iliaca is the facia that covers iliopsoas muscle, it is attached distally to fascia lata reaching the anterior thigh. The virtual space between iliopsoas muscle and the fascia iliaca is called fascia iliaca compartment, it is the distal continuation of the space between quadratus lumborum muscle and fascia. Three important nerves for sensory innervation of the thigh are located in this compartment, namely, obturator nerve, lateral femoral cutaneous nerve, and femoral nerve, a technique comparable to three in one nerve block. ${ }^{5}$ Landmark when performing FICB is the inguinal ligament. It can be achieved without ultrasound guidance with a characteristic ${ }^{2}$ pops felt, but with lower success rate. ${ }^{\mathbf{5}} \mathrm{A}$ higher rate of success (>85\%), and relatively quick fashion occur when performing it in real-time ultrasonic guidance. ${ }^{6}$ A supra-inguinal ultrasound guided approach has been described. It produces sensory block of the lateral, anterior, and medial aspects of the thigh. Moreover, it leads to more spread of local anesthetic in the cranial direction towards the lumbar plexus and the targeted nerves. The sufficient suggested volume to reach these nerves should be $40 \mathrm{ml} .{ }^{5}$

FICB can be delivered as either a single shot or a continuous infusion. The technique for continuous infusion catheter is like that for a single shot. Once the needle is in the correct position, a catheter can be advanced for 2-4 $\mathrm{cm}$. A bolus dose of local anesthetic (LA) is injected followed by either a continuous infusion and/or intermittent boluses of LA via an infusion pump. Infusion pumps can either be electronic or nonelectronic e.g. elastomeric pumps. ${ }^{7}$

FICB provides better analgesic effect compared to IV opioids for patient positioning during spinal anesthesia in femur fracture surgeries. ${ }^{8}$ Moreover, the fascia iliaca compartment catheter can be placed faster than femoral nerve catheter, but with longer time till onset of sensory block. ${ }^{3,9}$
The efficacy of bupivacaine, an amid local anesthetic drug, has been highlighted in various studies considering its availability and low price. Unfortunately, bupivacaine may display a delayed onset of action, ${ }^{4}$ and the use of dexmedetomidine as an adjuvant may solve this problem.

Dexmedetomidine, an $\alpha 2$ adrenergic receptor agonist, exerts its analgesic and sedative effects through its $\alpha 2$ receptor affinity. ${ }^{10} \mathrm{Li}$, et al. ${ }^{11}$ suggested that dexmedetomidine can increase the efficacy and prolong the duration of local anesthetic blockade.

It has been added to bupivacaine to decrease the onset time for sensory block during spinal, epidural, and brachial plexus blocks with good results. ${ }^{12,13,14}$

Although few randomized studies investigated the postoperative effect of dexmedetomidine as an adjuvant to LA in supra-inguinal FICB, there is little knowledge about its preoperative effect, and to our knowledge, no study has been investigated the effect of adding dexmedetomidine to bupivacaine in FICB as regard facilitating spinal anesthesia.

The study aimed to determine the efficacy of adding dexmedetomidine to bupivacaine while performing preoperative ultrasonic supra-inguinal FICB to provide analgesia for positioning femur fracture patients before spinal anesthesia. Our primary outcome is time to sensory block, while number of patients required analgesia during performance of spinal anesthesia, post-operative pain, duration till analgesic request, and 24 hours analgesic consumption are secondary outcomes.

\section{PATIENT AND METHODS}

Sample size was calculated in this study by using G*Power 3.1.9.2 program, Kiel, Germany; depending on our primary outcome (time to sensory block), in a previous study the time to sensory block in bupivacaine treated patient was $18.8 \pm 5.3 \mathrm{~min}$, in this study to detect at least $25 \%$ decrease in time to sensory block and a power of $85 \%(1-\beta)$, at $5 \%$ significance level $(\alpha)$, the minimum sample size was to study 24 patients in each group. With an increase of $10 \%(\approx 3$ patients $)$ for dropouts, so we investigated 30 patients in each group.

After local ethics committee approval and informed written consents from the patients this prospective double blinded randomized controlled study was conducted from 1 April 2019 to 31 March 2020 at Al-Azhar university hospitals. Sixty patients, American Society of Anesthesiologists (ASA) physical status I-III, aged from 50 to 70 years and weighting from 60 to $90 \mathrm{~kg}$ underwent femur fracture surgeries under spinal anesthesia were included in the study . 
Exclusion criteria were as follows: refusal of the patient to spinal anesthesia or to participate in the study, impaired renal or hepatic functions, patients with multiple trauma, obesity (Body Mass Index > 35 ), allergy to study drugs, local infection, previous surgery at the injection area, bleeding disorders, patients on anticoagulant therapy, addicts, an opioid analgesic prescription within the last $2 \mathrm{~h}$ before the operation, peripheral neuropathy, mental and psychiatric disorders.

Before operation, the written consent was taken, and the patient received instructions for using $(0-10)$ visual analogue scale (VAS) from $0=$ No pain to 10 $=$ worst imaginable pain

Using computer-generated randomization and sealed opaque envelope, patients were randomly assigned to one of the two equal groups (30 patients each) according to drug injected in FICB; Control group received $40 \mathrm{ml}$ bupivacaine $0.25 \%$, and Dexmedetomidine group received a mixture of dexmedetomidine $80 \mu \mathrm{g}$ in $40 \mathrm{ml}$ bupivacaine $0.25 \%$.

In the preoperative holding area, patients were premedicated with midazolam $(0.02 \mathrm{mg} / \mathrm{kg})$ after application of standard monitoring and $3 \mathrm{~L}$ nasal $\mathrm{O}_{2}$, then FICB were given to all patients.

Ultrasound-guided block was performed in supine position, inguinal ligament was identified, and crease area was sterilized using povidone iodine. Using a 5$12 \mathrm{MHz}$ linear probe (SonoSite ${ }^{\mathrm{TM}} \mathrm{M}$-Turbo, USA), started on the inguinal crease parallel to the inguinal ligament, and after identification of femoral artery (Figure: 1), a little movement of the probe laterally till iliopsoas muscle was specified as a hypo echo part in lateral to the artery and femoral nerve. It is covered by a hyperechoic fascia, which separates the muscle from the subcutaneous tissue (Figure: 2).

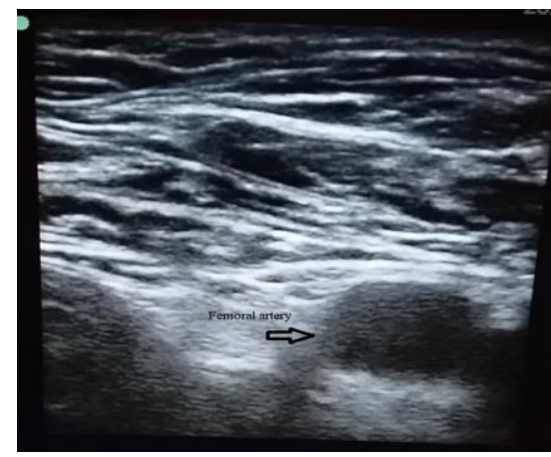

Figure 1: Femoral artery

After dermal anesthesia with $2 \mathrm{cc}$ lidocaine $1 \%$ at the place of needle, using the in-plane technique a $22 \mathrm{G} / 80 \mathrm{~mm}$ insulated echogenic block needle was inserted and advanced towards the fascia iliaca and iliopsoas muscle. Confirming the passage of the needle through the fascia iliaca using fascial click and $2 \mathrm{~mL}$ of saline. LA was injected between fascia iliaca and iliopsoas muscle, we used ultrasound guided hydro dissection technique. Through this hydro dissection, the needle was moved towards the space created by the injected LA. Time just after finishing injection was considered 0 point, and all times were calculated from it.

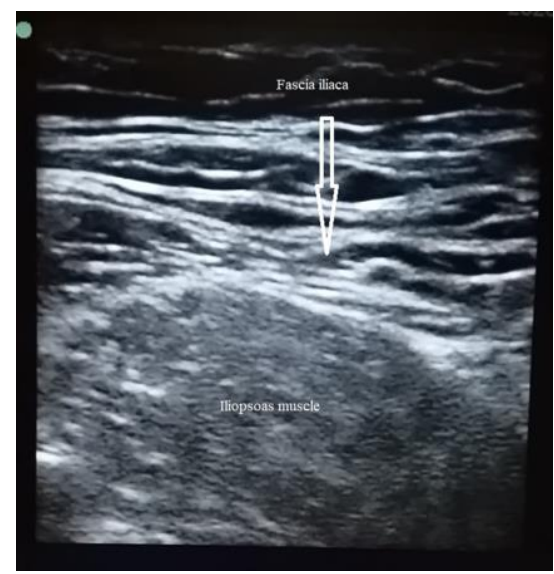

Figure 2: Iliopsoas muscle and fascia Iliaca

The responsible anesthesiologist and the patient were unaware of the nature of drug in each syringe and master codes were stored by personnel who did not participate in observation.

After block, IV $10 \mathrm{ml} / \mathrm{kg}$ Ringer's solution was given to the patient over 20 minutes then trans $\neg$ ferred to the operating room.

Time to sensory block was defined as the time till loss of cold sensation, or the patient express dramatic decrease in pain at fracture site.

In operating room; anesthesia administration was performed similarly for all patients utilizing a standard protocol; patients were positioned in the sitting position, the back was prepared using povidone iodine, and the area was draped with a sterile towel, lumbar puncture was performed through the L3-4 interspace using 25G spinal needle, and $12.5 \mathrm{mg}$ heavy bupivacaine $0.5 \%$ was injected.

IV fentanyl $25 \mu \mathrm{g}$ was administered if patient expressed moderate or severe pain during movement and positioning for spinal and could be repeated after 3 minutes till the patient expressed mild or no pain. Number of patients required fentanyl, total fentanyl requirement and the maximum VAS were recorded and compared.

For all patients, Paracetamol 1 gram was given IV infusion over 30minutes, 6 hours after spinal block, and repeated every 8 hours.

Postoperative; VAS was recorded at 2, 3, 4, 6, 8, 12, 18 and 24 hours. Pethidine $20 \mathrm{mg}$ was administered IV as rescue analgesic if VAS pain score was more than 3 or the patient asked for analgesia and can be repeated after 20 minutes till $\mathrm{VAS} \leq 3$. The time to first analgesic request and 24 hours total analgesic requirement were recorded and compared. Incidence 
of hematoma, systemic drug toxicity or any other complication were also recorded.

Statistical analysis: Data was analyzed using SPSS version 17 statistical software (SPSS, Chicago, IL, USA). Data were presented as mean+SD, Median and Inter Quartile Range (IQR), or number of patients. Student t-test were used for comparison of parametric data. Mann-Whitney test was used to compare non-parametric data. Chi-square and Fischer exact tests were used for comparison between number and frequencies. $\mathrm{P}<0.05$ was considered significant.

\section{RESULTS}

Patients' characteristics and duration of surgery were comparable between the two groups (Table 1).

Time to sensory block was significantly shorter in .Dexmedetomidine group compared to Control

Pain during positioning for spinal anesthesia was significantly lower in Dexmedetomidine group, but there were no significant differences as regards number of patients required fentanyl or total fentanyl among patients required fentanyl in both groups (Table 2).

Pain score was significantly lower after 6 and 8 hours postoperatively in Dexmedetomidine, otherwise, both groups were comparable (Table 3 ).
Time to 1 st analgesic request was significantly longer, and 24 hours analgesic consumption was significantly less in Dexmedetomidine patients compared to Control (Table 4).

No incidence of hematoma or systemic drug toxicity in our patients.

\section{DISCUSSION}

Spinal anesthesia is the routinely used technique for patients with femur fracture. Preoperative movement and positioning for spinal anesthesia is associated with severe and sometimes intolerable pain, however, pain relief for this situation is usually inadequate, even no analgesia is given for positioning in some patients. ${ }^{3}$ Providing effective analgesia for these patients is complicated by advanced age and concomitant comorbidities, also inadequately treated pain has several consequences for geriatric patients with fractures. ${ }^{3,7}$

In orthopedic operations, nerve block techniques are preferred for pain control either pre or postoperative. ${ }^{4}$ These techniques can be quick and safe to improve analgesia and patient satisfaction. ${ }^{7}$ Nerve blocks not only improve perioperative patient comfort and reduce pain that exaggerated by movements but also increase the ease, success rate, and decrease time for performing spinal anesthesia.

\begin{tabular}{|c|c|c|c|c|}
\hline & & $\begin{array}{l}\text { Dexmedetomidine Group } \\
\qquad(\mathrm{n}=30)\end{array}$ & $\begin{array}{l}\text { Control Group } \\
\qquad(\mathrm{n}=30)\end{array}$ & P-value \\
\hline Age (years) & & $58.9 \pm 5.9$ & $60 \pm 7.6$ & $>0.05$ \\
\hline Weight (kg) & & $79.9 \pm 7.3$ & $78.2 \pm 8.3$ & $>0.05$ \\
\hline BMI & & $29.9 \pm 3.1$ & $30.3 \pm 3.3$ & $>0.05$ \\
\hline \multirow{3}{*}{ ASA } & $\mathrm{I}$ & 7 & 6 & \multirow{3}{*}{$>0.05$} \\
\hline & II & 12 & 11 & \\
\hline & III & 11 & 13 & \\
\hline \multirow[t]{2}{*}{ Sex } & Male & 17 & 14 & \multirow{2}{*}{$>0.05$} \\
\hline & Female & 13 & 16 & \\
\hline \multicolumn{2}{|c|}{ Duration of Surgery (min) } & $90.8 \pm 14.3$ & $95.8 \pm 12.8$ & $>0.05$ \\
\hline
\end{tabular}

Table 1: Patients' characteristics and duration of surgery (Data are expressed as mean \pm SD and number)

\begin{tabular}{|c|c|c|c|}
\hline & $\begin{array}{c}\text { Dexmedetomidine Group } \\
(\mathrm{n}=30)\end{array}$ & $\begin{array}{c}\text { Control Group } \\
(\mathrm{n}=30)\end{array}$ & P-value \\
\hline Time to sensory block $(\mathrm{min})$ & $14.4 \pm 2.1$ & $17.3 \pm 2.3$ & $<0.001$ \\
\hline Number of patients required fentanyl & 4 & 6 & $>0.05$ \\
\hline Total fentanyl $(\mu \mathrm{g})$ & $31.25 \pm 12.5$ & $37.5 \pm 13.69$ & $>0.05$ \\
\hline VAS during movement & $2(1-3)$ & $3(3-4)$ & $<0.05$ \\
\hline
\end{tabular}

Table 2: Block characteristics and fentanyl requirements (Data are expressed as mean $\pm \mathrm{SD}$, median [Inter Quartile Range], or number) 


\begin{tabular}{|c|c|c|c|}
\hline & $\begin{array}{c}\text { Dexmedetomidine Group } \\
(\mathrm{n}=30)\end{array}$ & $\begin{array}{c}\text { Control Group } \\
(\mathrm{n}=30)\end{array}$ & P-value \\
\hline 2 hours & $0(0-0)$ & $0(0-0)$ & $>0.05$ \\
\hline 3 hours & $0(0-0)$ & $0(0-0)$ & $>0.05$ \\
\hline 4 hours & $0(0-0)$ & $0(0-1)$ & $>0.05$ \\
\hline 6 hours & $0(0-1)$ & $3(2-4)$ & $<0.001$ \\
\hline 8 hours & $1(1-2)$ & $4(3-5)$ & $<0.001$ \\
\hline 12 hours & $4(3-5)$ & $5(4-6)$ & $>0.05$ \\
\hline 18 hours & $5(4-5)$ & $5(4-6)$ & $>0.05$ \\
\hline 24 hours & $3(3-4)$ & $2-5)$ & $>0.05$ \\
\hline
\end{tabular}

Table 3: Postoperative pain score (Data are expressed as median (Inter Quartile Range)

\begin{tabular}{|c|c|c|c|}
\hline & $\begin{array}{c}\text { Dexmedetomidine Group } \\
(\mathrm{n}=30)\end{array}$ & $\begin{array}{c}\text { Control Group } \\
(\mathrm{n}=30)\end{array}$ & P-value \\
\hline Time to 1st analgesic request (hour) & $8.21 \pm 1.16$ & $5.73 \pm 1.12$ & $<0.001$ \\
\hline 24 hours analgesic consumption $(\mathrm{mg})$ & $58.7 \pm 21.6$ & $74 \pm 24.7$ & $<0.05$ \\
\hline
\end{tabular}

Table 4: Time to first analgesia and 24 hours analgesic consumption (Data are expressed as mean \pm SD)

Guidelines from United Kingdom National Institute for Health and Clinical Excellence considered nerve block to provide analgesia and decrease narcotic dosage. ${ }^{3}$ Also, the American Academy of Orthopedic Surgeons (AAOS) clinical practice guidelines on management of hip fractures in elderly provide a strong recommendation for regional blocks to improve preoperative pain control. ${ }^{7}$

There is a high-quality evidence that regional blocks reduce pain on movement within 30 minutes of block placement and moderate quality evidence for decreasing time to 1 st mobilization after surgery. In spite of benefits of these procedures and national guidelines recommending their use, regional nerve blocks are not being used on a regular basis. ${ }^{7}$

The ideal pre-operative regional nerve block for patients with femur fractures must be simple, easily accessed and can performed in the supine position without any patient movement. The primary approaches include quadratus lumborum block, FNB and FICB. ${ }^{7,15}$

FICB can be easily performed and has been used as a good solution for managing femur fracture pain in emergency departments. ${ }^{16}$ Evans, et al. ${ }^{2}$ reported prehospital administration of FICB for patients with suspected fractures at the scene of trauma, using the landmark technique with good results. Hards, et al. ${ }^{17}$ in their systematic review concluded that FICB is appropriate technique for prehospital pain management, with a high success rate and few adverse effects. It was concluded that it is easily learned and effective procedure for preoperative pain. ${ }^{18}$ Although some studies did not outline the training program for junior doctors learning to perform FICB, the authors noted that the learning curve was relatively easy. ${ }^{7}$
Fascia iliaca compartment is the distal continuation with the space between quadratus lumborum muscle and fascia. Anterior approach quadratus lumborum block cab be considered for postoperative pain management in proximal femur fractures. But as regard preoperative analgesia, FICB is superior to quadratus lumborum block because the later needs patient positioning. ${ }^{9}$

Jain, et al. ${ }^{3}$ compared both FNB with FICB in patients with femur fractures to reduce pain during positioning for spinal anesthesia, they proved that FICB has delayed onset of action in reducing pain during positioning (sitting) for spinal anesthesia.

Bupivacaine has been used in local anesthesia techniques in various studies, and its efficacy in different concentrations has been studied. Some studies had revealed that, usage of $0.5 \%$ bupivacaine for peripheral nerve blocks provides longer duration of postoperative analgesia for lower limb surgeries than $0.25 \%$ bupivacaine. Another study finding showed that, either $0.5 \%$ bupivacaine or $0.25 \%$ is accompanied by adequate analgesia with no difference in the duration of action. however, patients receiving $0.5 \%$ bupivacaine had lower satisfaction due to occurrence of numbness, weakness, and delay in the walking. ${ }^{4}$

Also, bupivacaine may demonstrate a long time till onset of analgesia, 4 evidence proved pain reduction on movement may be delayed till 30 minutes after FICB. ${ }^{7}$ There has been growing attention in using local anesthetic (LA) adjuvants to accelerate the onset of action and prolong the analgesic effect of LA. ${ }^{14,19}$

Multiple studies have shown that using dexmedetomidine as an adjuvant to LA in peripheral nerve techniques enhance the onset of action, 
increase the duration of analgesia, and decrease postoperative analgesic intake. ${ }^{20,21}$

As regard FICB, Sabra, et al ${ }^{15}$ added dexmedetomidine to ropivacaine in order to study its postoperative analgesic effect in hip arthroplasty, they reported that combining ropivacaine and dexmedetomidine significantly decrease pain scores and ${ }^{24}$ hours analgesic requirement as opposed to solely using ropivacaine.

Sivakumar, et al. ${ }^{19}$ also added dexmedetomidine to LA in FICB for femur surgeries but with general anesthesia. While $\mathrm{Li}$, et al. ${ }^{11}$ studied the effect of adding dexmedetomidine to ropivacaine in FICB for knee arthroscopy, both studies proved decrease pain scores and total analgesic requirement. To be noted, in these studies, all injections were given after anesthesia that the time for analgesia onset could not be assessed. ${ }^{\mathbf{1 5 , 1 9}}$

Mechanism of action of dexmedetomidine on peripheral nerves is not clear. Suggested mechanisms include direct drug effect on the nerves and absorption into systemic circulation causing central effect. ${ }^{19}$

No recorded adverse effects in our patients. On the other hand, FICB can reduce narcotic related side effects and this was proved by many studies ${ }^{15,22}$

Limitations of our study include lack of pain measurement in different motor states, and there was no long-term follow-up as hospital stay and longterm neurological complications were not assessed.

We recommend further studies to know that effect of dexmedetomidine on pain in various motor states, and to compare its effect with IV dexmedetomidine.

\section{CONCLUSION}

Addition of dexmedetomidine to bupivacaine is effective in enhancing onset of fascia iliaca block and decreasing severity of pain during positioning for spinal anesthesia in fracture femur patients, with added longer duration of the analgesia and reduced postoperative analgesic requirements.

\section{REFERENCES}

1. Wu SC, Rau CS, Kuo SCH, et al. The influence of ageing on the incidence and site of trauma femoral fractures: a cross-sectional analysis. BMC Musculoskelet Disord. 2019; 20(1): 413.

2. Evans BA, Brown A, Bulger J, et al. Paramedics' experiences of administering fascia iliaca compartment block to patients in South Wales with suspected hip fracture at the scene of injury: results of focus groups. BMJ Open. 2019; 9(2): 026073 .
3. Jain N, Mathur PR, Patodi V, et al. A comparative study of ultrasound-guided femoral nerve block versus fascia iliaca compartment block in patients with fracture femur for reducing pain associated with positioning for subarachnoid block. Indian J Pain. 2018; 32:150-4.

4. Rahimzadeh P, Imani F, Sayarifard A, et al. Ultrasound-guided fascia iliaca compartment block in orthopedic fractures: Bupivacaine $0.2 \%$ or 0.3\%?. Med J Islam Repub Iran. 2016 24; 30:433.

5. Ling C, Liu XQ, Li YQ, et al. Ultrasound-guided fascia iliaca compartment block combined with general anesthesia for amputation in an acute myocardial infarction patient after percutaneous coronary intervention: A case report. World $J$ Clin Cases. 2019; 7(17): 2567-72.

6. Bullock WM, Yalamuri SM, Gregory SH, et al. Ultrasound-Guided Suprainguinal Fascia Iliaca Technique Provides Benefit as an Analgesic Adjunct for Patients Undergoing Total Hip Arthroplasty. J Ultrasound Med 2017; 36: 433-8.

7. Scala VA, Lee LSK and Atkinson RE. Implementing Regional Nerve Blocks in Hip Fracture Programs: A Review of Regional Nerve Blocks, Protocols in the Literature, and the Current Protocol at The Queen's Medical Center in Honolulu, HI. Hawaii J Health Soc Welf. 2019;78:11-15.

8. Madabushi R, Rajappa GC, Thammanna PP, et al. Fascia iliaca block vs intravenous fentanyl as an analgesic technique before positioning for spinal anesthesia in patients undergoing surgery for femur fractures-a randomized trial. $J$ ClinAnesth. 2016; 35: 398-403.

9. Plečko M, Bohaček I, Tripković B, et al.. Applications and critical evaluation of fascia iliaca compartment block and quadratus lumborum block for orthopedic procedures. Acta Clin Croat. 2019; 58(1): 108-13.

10. Abotaleb UI, Abdalla AM, Abdelrahman AS, et al. Dexmedetomidine versus granisetron for the management of postspinal shivering. Ain-Shams $J$ Anaesthesiol. 2016; 9: 517-23

11. Li Y, Geng J, Wen L, et al. Postoperative analgesia with ropivacaine and dexmedetomidine for ultrasound-guided fascia iliaca compartment block after arthroscopic knee surgery. Saudi $J$ Anaesth. 2019; 13(2):100-5.

12. Gousheh M, Akhondzadeh R, Rashidi M, et al. Comparison of Dexmedetomidine and Morphine as Adjuvants to Bupivacaine for Epidural Anesthesia in Leg Fracture Surgery: A Randomized Clinical Trial. Anesth Pain Med. 2019; 9(4):e91480.

13. Avula RR, Vemuri NN and Puthi S. Ultrasound- guided subclavian perivascular brachial plexus block using $0.5 \%$ bupivacaine with dexmedetomidine as an adjuvant: A prospective randomized controlled trial. Anesth Essays Res. 2019; 13: 615-9.

14. Liu $X$, Zhang $X$, Wang $X$, et al. Comparative evaluation of intrathecal bupivacaine alone and bupivacaine combined with dexmedetomidine in cesarean section using spinal anesthesia: a metaanalysis. J Int Med Res. 2019; 47(7): 2785-99. 
15. Sabra MM, Abdalla $M$ and Abdelrahman AS. Efficacy of ultrasound-guided fascia iliaca compartment block with ropivacaine and dexmedetomidine for postoperative analgesia in hip arthroplasty. Al-Azhar Assiut Med J 2019;17:378-84.

16. Wilson J, Maloney $\mathrm{K}$, Bookman $\mathrm{K}$, et al. Training Emergency Physicians in Ultrasound guided Fascia Iliaca Compartment Blocks: Lessons in Change Management. Cureus. 2019; 11(5): 4773.

17. Hards M, Brewer A, Bessant G, et al. Efficacy of Prehospital Analgesia with Fascia Iliaca Compartment Block for Femoral bone Fractures: A Systematic Review. Prehosp Disaster. Med. 2018; 33(3): 299-307.

18. Hao J, Dong B, Zhang J, et al. Pre-emptive analgesia with continuous fascia iliaca compartment block reduces postoperative delirium in elderly patients with hip fracture. Saudi Med J. 2019; 40(9): 901-6.

19. Sivakumar RK, Panneerselvam S, Cherian A, et al. intravenous dexmedetomidine as an adjunct to bupivacaine in ultrasound guided fascia iliaca compartment block for femur surgeries: A randomised control trial. Indian J Anaesth. 2018; 62(11): 851-7.

20. Andersen JH, Grevstad U, Siegel H, et al. Does Dexmedetomidine Have a Perineural Mechanism of Action When Used as an Adjuvant to Ropivacaine?: A Paired, Blinded, Randomized Trial in Healthy Volunteers. Anesthesiology. 2017;126(1): 66-73.

21. Chinnappa J, Shivanna S, Pujari VS, et al. Efficacy of dexmedetomidine with ropivacaine in supraclavicular brachial plexus block for upper limb surgeries. J Anaesthesiol Clin Pharmacol. 2017; 33(1): 81-5.

22. Kumar D, Hooda S, Kiran S and Devi J. Analgesic efficacy of ultrasound guided FICB in patients with hip fracture. J Clin Diagn Res. 2016;10: 13-16. 